\title{
SUL TEOREMA DI ESISTENZA DELLE FUNZIONI ABELIANE
}

Come è ben noto, condizione necessaria e sufficiente perchè il quadro di numerì

$$
\left|\begin{array}{cccc}
\omega_{11} & \omega_{12} & \ldots & \omega_{1,2 p} \\
\omega_{21} & \omega_{22} & \ldots & \omega_{2,2 p} \\
\cdots & \ldots & \ldots & \ldots \\
\omega_{p 1} & \omega_{p 2} & \ldots & \omega_{p, 2 p}
\end{array}\right|
$$

possa pensarsi come la tabella di un sistema di periodi indipendenti per una funzione abeliana a $p$ variabili, cioè per una fnnzione meromorfa di $p$ variabili $u_{1}, u_{2}, \ldots, u_{p} 2 p$ volte periodica è :

A) che esistano fra le $\omega_{j k} \frac{1}{2} p(p-1)$ relazioni del tipo

$$
\sum_{r, s}^{1 \ldots 2 p} c_{r s} \omega_{j r} \omega_{k s}=0 \quad(j, k=1,2, \ldots, p ; j<k)
$$

essendo le $c_{r s}$ dei numeri interi legati dalla relazione

$$
c_{r s}+c_{s r}=0
$$

$B)$ che indicati con $\Omega_{j}(j=1,2, \ldots, 2 p)$ i $2 p$ periodi di una combinazione lineare omogenea qualunque

$$
\lambda_{1} u_{1}+\lambda_{2} u_{2}+\ldots+\lambda_{p} u_{p}
$$

delle variabili $u_{1}, u_{2}, \ldots, u_{p}$, corrispondenti ai $2 p$ sistemi di periodi costituenti la tabella (1), e posto

$$
\Omega_{j}=\xi_{j}+i \eta_{j} \quad(i=\sqrt{-1})
$$


con le $\xi_{j}$ e $\eta_{j}$ reali, si abbia sempre

o sempre (1)

$$
\sum_{r, s}^{1 \ldots 2 p} c_{r s} \xi_{r} \eta_{s}>0
$$$$
\sum_{r, s}^{1 \ldots 2 p} c_{r s} \xi_{r} \eta_{s}<0
$$

Di queste due condizioni la $B$ ) non è molto maneggevole e nou sempre è adatta alle applicazioni; e infutti i signori BAGNERA e DF FRanchis nelle loro belle ricerche sulle superficie iperellittiche $\left({ }^{2}\right)$ raggiunsero una grande facilitazione nei loro procedimenti allorchò nella seconda delle due Memorie citate a piè di pagina osservarono che la condizione $B$ ), per il caso $p=2$, poteva esser trasformata in un'altra rappresentata da una diseguaglianza contenente soltanto, $\Theta$ in fattori distiuti, le $c_{r s}$ e le $\tilde{\omega}_{j k} ; 0$, più precisamente, le $c_{r s}$ ele parti reali $\theta$ i coefficienti dell'immaginario delle $\omega_{j k}$.

Ebbene noi vogliamo dimostrare in questa Nota che il teorema dei signori Bagnera e DE Franchis può estendersi al caso di $p(\geq 2)$ qualunque; e la generalizzazione appare tanto più interessante, in quanto che solo per $p=2$ possono essere utilizzati, come appunto hanno fatto i signori BAGNERA O DE FRANCHIS, con ragionamento semplice ed elegante, taluni classici risultati del FrobENiUs.

La proposizione che noi vogliamo stabilire è la seguente.

Si ponga

$$
\omega_{j k}=\alpha_{j k}+i \beta_{j k} \quad(i=\sqrt{-1})
$$

con le $\alpha_{j k}$ e $\beta_{j k}$ reali $e$ si considerino $i$ due determinanti

$$
\left|\begin{array}{lllll}
0 & c_{12} & c_{13} & \ldots & c_{1,2 p} \\
c_{21} & 0 & c_{23} & \ldots & c_{2,2 p} \\
\cdots & \ldots & \ldots & \ldots & \ldots \\
c_{2 p, 1} & c_{2 p, 2} & c_{2 p, 3} & \ldots & 0
\end{array}\right|
$$

(1) Ordinariamente si parla soltanto della diseguaglianza (5), poiohè, nve nocorra, cambiando il segno a tutte lo $o_{r a}$ si prio ottenere ohe la (6) si oonverta nella (5).

(2) G. Bagnera o M. DE Franchis: a) Le superficie algebriche le quali ammettono una rappresentazione parametrica mediante funzioni iperellittiohe di due argomenti [Memorie di Matematica e di Fisioa della Societa Italiana delle Soienze, serie III, tomo XV (1908), pp. 251-343]; b) Le nombre @ de M. PICARD pour les surfaces hyperelliptiques et pour les surfases irrégulières de genre zéro [Rendiconti del Circolo Matematico di Palermo, tomo XXX (20 senestre 1910), pp. 185-235]. 


$$
\left|\begin{array}{ccccc}
\alpha_{11} & \alpha_{12} & \alpha_{13} & \ldots & \alpha_{1,2 p} \\
\alpha_{21} & \alpha_{22} & \alpha_{23} & \ldots & \alpha_{2,2 p} \\
\ldots & \ldots & \ldots & \ldots & \ldots \\
\alpha_{p 1} & \alpha_{p 2} & \alpha_{p 3} & \ldots & \alpha_{p, 2 p} \\
\beta_{11} & \beta_{12} & \beta_{13} & \ldots & \beta_{1,2 p} \\
\beta_{21} & \beta_{22} & \beta_{23} & \ldots & \beta_{2,2 p} \\
\ldots & \ldots & \ldots & \ldots & \ldots \\
\beta_{p 1} & \beta_{p 2} & \beta_{p 3} & \ldots & \beta_{p, 2 p}
\end{array}\right|
$$

dei quali il primo è, per quanto sappiamo, emisimmetrico.

Poi, indicata con

$$
j_{1}, j_{2}, \ldots, j_{2 l} \quad(1 \leq l \leq p)
$$

una serie di indici distinti estratti dalla successione

$$
1,2, \ldots, 2 p
$$

e ordinati per grandezza crescente, si chiami

$$
P_{j_{1} j_{2} \ldots j_{2 l}}
$$

lo pfaffino del determinante emisimmetrico formato dalle righe e dalle colonne del determinante (7) aventi $i n$. d'ordine $j_{1}, j_{2}, \ldots, j_{2 l} ;$ e

$$
\delta_{j_{1} j_{2} \ldots j_{2 l}}
$$

il minore che si ottiene da (8) considerando le righe e le colonne coi n. d'ordine rispettivi $1,2, \ldots, l, p+1, p+2, \ldots, p+l ; e j_{1}, j_{2}, \ldots$ $\ldots, j_{2 l}$.

Se si pone

$$
\Delta_{l}=(-1)^{\frac{l(l-1)}{2}} \sum P_{j_{1} j_{2} \ldots j_{2 l}} \delta_{j_{1} j_{2} \ldots j_{2 l}} \quad(l=1,2, \ldots, p),
$$

dove il sommatorio si intende esteso a tutte le possibili serie $j_{1}, j_{2}, \ldots$ $\ldots, j_{2 l}$ soddisfacenti alle restrizioni indicate più sopra, la condizione B) può esser sostituita dalle seguenti $p-1$ diseguaglianze

$$
\Delta_{2}>0, \Delta_{1} \Delta_{3}>0, \Delta_{4}>0, \Delta_{1} \Delta_{5}>0, \ldots ;
$$


delle quali l'ultima è

$$
\Delta_{p}>0 \quad \text { o } \quad \Delta_{1} \Delta_{p}>0
$$

secondo che $p$ è pari o dispari $\left({ }^{1}\right)$.

1. Indicato con $\mu$ un qualsivoglia numero complesso, chiamiamo $\bar{\mu}$ il numero complesso coniugato; allora da

si trae

$$
\Omega_{j}=\xi_{j}+i \eta_{j}
$$

e quindi

$$
\bar{\Omega}_{j}=\xi_{j}-i \eta_{j}
$$

$$
\xi_{j}=\frac{1}{2}\left(\Omega_{j}+\bar{\Omega}_{j}\right), \quad \eta_{j}=\frac{1}{2 i}\left(\Omega_{j}-\bar{\Omega}_{j}\right)
$$

Segue che

ossia che

$$
\sum_{r, s}^{1 \ldots 2 p} c_{r s} \xi_{r} \eta_{s}=\frac{1}{4 i} \sum_{r, s}^{1 \ldots 2 p} c_{r s}\left(\Omega_{r}+\bar{\Omega}_{r}\right)\left(\Omega_{s}-\bar{\Omega}_{s}\right)
$$

$$
\sum_{r, s}^{1 \ldots 2 p} c_{r s} \xi_{r} \eta_{s}=-\frac{1}{2 i} \sum_{r, s}^{1 \ldots 2 p} c_{r s} \Omega_{r} \bar{\Omega}_{s}
$$

una volta che, in base all'ipotesi (3) fatta sulle $c_{r s}$, si ha evidentemente

$$
\sum_{r, s}^{1 \ldots 2 p} c_{r s} \Omega_{r} \Omega_{s}=\sum_{r, s}^{1 \ldots 2 p} c_{r s} \bar{\Omega}_{r} \bar{\Omega}_{s}=0
$$

e

$$
\sum_{r, s}^{1 \ldots 2 p} c_{r s} \bar{\Omega}_{r} \Omega_{s}=-\sum_{r, s}^{1 \ldots 2 p} c_{r s} \Omega_{r} \bar{\Omega}_{s}
$$

(1) Di questo teorema sono in possesso tin dal gennaio a. s. ma la via ohe allora arevo scelta per dimostrarlo non solo rendeva necessarie delle ricerche un pò minute d'indole aritmetica, di cui una parte è stata racoolta nella mia Nota: Osservazioni varie sulla teoria delle sostiluzioni e sulle partizioni dei numeri interi in numeri interi [Rendiconti del Circolo Matematioo di Palermo, t. XXXVI (20 semestre 1913), pp. 163-170], ma portava anohe trnto in Iungo la dimostrazione che ho preferito attendere a pubblioare il teorema quando fossi riusoito a darne una dimostrazione più semplice. E quella che pubblico ora mi sembra la più di. retta e la più semplice possibile.

Osservisi, per il oonfronto di qnesto teorema con quello dei signori BaGNera e DE Franchis, ohe essi indicano con $\delta$ eid che seoondo le nostre notazioni à da indicarsi con $-\boldsymbol{P}_{1234}$. 
I'altro canto

dunque

$$
\Omega_{r} \bar{\Omega}_{s}=\sum_{j, k}^{1 \ldots p} \omega_{j r} \bar{\omega}_{k s} \lambda_{j} \bar{\lambda}_{k}
$$

$$
\sum_{r, s}^{1 \ldots 2 p} c_{r s} \xi_{r} \eta_{s}=-\frac{1}{2 i} \sum_{r, s}^{1 \ldots 2 p} \sum_{j, k}^{1 \ldots p} c_{r s} \omega_{j r} \overline{\vec{\omega}}_{k s} \lambda_{j} \bar{\lambda}_{k} .
$$

Poniamo

cioè

$$
\sum_{r, s}^{1 \ldots 2 p} c_{r s} \xi_{r} \eta_{s}=\sum_{j, k}^{1 \ldots p} A_{j k} \lambda_{j} \bar{\lambda}_{k}
$$

$$
A_{j k}=-\frac{1}{2 i} \sum_{r, s}^{1 \ldots 2 p} c_{r s} \omega_{j r} \bar{\omega}_{k s}
$$

sarà

$$
A_{k j}=-\frac{1}{2 i} \sum_{r, s}^{1 \ldots 2 p} c_{r s} \omega_{k r} \bar{\omega}_{j s}=+\frac{1}{2 i} \sum_{r, s}^{1 \ldots 2 p} c_{r s} \bar{\omega}_{j r} \omega_{k s}=\bar{A}_{j k}
$$

quindi l'espressione

$$
\sum_{r, s}^{1 \ldots 2 p} c_{r s} \xi_{r} \eta_{s}
$$

non è altra cosa che una forma Hermitiana nelle variabili $\lambda_{1}, \lambda_{2}, \ldots$ $\ldots, \lambda_{p}$ (e nelle loro coniugate $\bar{\lambda}_{1}, \bar{\lambda}_{2}, \ldots, \bar{\lambda}_{p}$ ).

2. In base a questa osservazione, la condizione B) può esser sostituita dalle diseguaglianze atte ad esprimere che la forma Hermitiana

$$
\sum_{j, k}^{1 \ldots p} A_{j k} \lambda_{j} \overline{\lambda_{k}}
$$

è definita (positiva o negativa).

Ora se si poue

$$
\varepsilon_{h}=\left|\begin{array}{cccc}
A_{11} & A_{12} & \ldots & A_{1 h} \\
A_{21} & A_{22} & \ldots & A_{2 h} \\
\ldots & \ldots & \ldots & . \\
A_{h 1} & A_{h 2} & \ldots & A_{h h}
\end{array}\right| \quad(h=1,2, \ldots, p)
$$

la forma Hermitiana (10) è defiuita positiva quaudo (e solo quando)

$$
\varepsilon_{1}>0, \varepsilon_{2}>0, \varepsilon_{3}>0, \ldots, \varepsilon_{p}>0
$$


ed è definita uegativa quando (e solo quando) $\left(^{1}\right.$ )

$$
\varepsilon_{1}<0, \varepsilon_{2}>0, \varepsilon_{3}<0, \ldots,(-1)^{p} \varepsilon_{p}>0
$$

duuque perchè essa sia defuita (positiva o negativa) occorre e basta $\operatorname{ch} \theta \operatorname{sia}\left({ }^{2}\right)$

$$
\varepsilon_{2}>0, \varepsilon_{1} \varepsilon_{3}>0, \varepsilon_{4}>0, \varepsilon_{1} \varepsilon_{5}>0, \ldots
$$

Segue che la nostra proposizione sarà dimostrata appena si sia futto vedere che

$$
\varepsilon_{h}=\Delta_{h} \quad(h=1,2, \ldots, p)
$$

3. Il ragionamento che sard fatto nel $\mathrm{n}$. successivo per limostrare che $\varepsilon_{h}=\Delta_{h}$ è indipendente da qualsiasi ipotesi particolare su $h$; pure, per chiarirne l'essenza, è bene trattar prima a parte $i$ casi di $h=1$ ed $h=2$.

Per $h=1$ si ba :

$$
\varepsilon_{1}=A_{11}=-\frac{1}{2 i} \sum_{r, s}^{1 \ldots 2 p} c_{r \cdot s} \omega_{1 r} \bar{\omega}_{1 s}=-\frac{1}{2 i} \sum_{r, s}^{1 \ldots 2 p} c_{r s}\left(\omega_{1 r} \bar{\omega}_{1 s}-\omega_{1 s} \bar{\omega}_{1 r}\right)
$$

dove l'apice apposto al simbolo dell'ultimo sommatorio sta ad indicare che $r$ ed $s$ variano fra $1,2, \ldots, 2 p$ in modo che sia sempre soddisfutta la diseguaglianza $r<s$.

Ora

dunque

$$
\omega_{1 r} \bar{\omega}_{1 s}-\omega_{1 s} \bar{\omega}_{1 r}=-2 i\left(\alpha_{1 r} \beta_{1 s}-\alpha_{1 s} \beta_{1 r}\right)
$$

$$
\varepsilon_{1}=\sum_{r, s}^{1 \ldots 2 p} c_{r s}^{\prime}\left(\alpha_{1 r} \beta_{1 s}-\alpha_{1 s} \beta_{1 r}\right)
$$

(1) Per quanto mi risulta questo teorema non à stato mai esplicitamente ennnciato; conunque fra le tante dimostrazioni ohe potrebbero darsene mi perwetto di richiamare l'attenzione del lettore eu quella contenuca nella mia Nota: Sopra una certa classe di determinanti e sulle forme Hermiliane, cho comparira in uno dei prossimi fuseicoli del Giornale di Matematiohe di Battaglini.

$(2)$ Non in intile far avvertire obe per il caso $p=2$ la condizione

comprende l'altra espressa da

$$
\varepsilon_{2}>0
$$

Infatti

$$
\varepsilon_{1} \neq 0 \text {. }
$$

$$
\varepsilon_{2}=A_{11} A_{22}-A_{12} A_{21}=\varepsilon_{1} A_{22}-A_{12} \bar{A}_{12}
$$

ed $A_{12} \bar{A}_{12}$ non pпò esser negativo. 
Ma il secondo membro di questu eguaglianza quando si adoperino i simboli definiti nell'introduzione può scriversi

quindi è chiaro che

$$
\sum P_{r s} \delta_{r s}
$$

$$
\varepsilon_{1}=\Delta_{1}
$$

Passiamo al caso $h=2$.

Qui si ha, col solito significato del simbolo $\Sigma^{\prime}$,

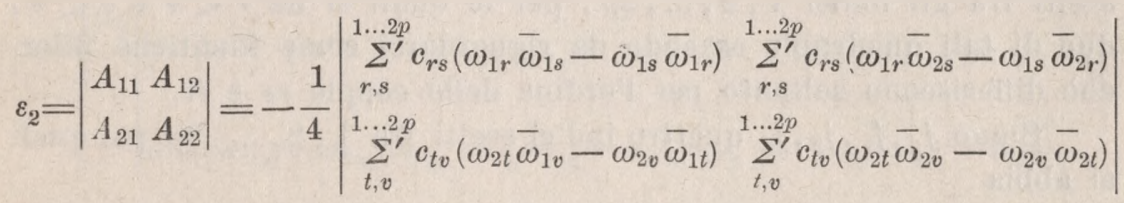

$$
\begin{aligned}
& =-\frac{1}{4} \sum_{r, s}^{1 \ldots 2 p} \sum_{t, v}^{1 \ldots 2 p} c_{r s} c_{t v}\left|\begin{array}{l}
\omega_{1 r} \bar{\omega}_{1 s}-\omega_{1 s} \bar{\omega}_{1 r} \omega_{1 r} \bar{\omega}_{2 s}-\omega_{1 s} \bar{\omega}_{2 r} \\
\omega_{2 t} \bar{\omega}_{1 v}-\omega_{2 v} \bar{\omega}_{1 t} \omega_{2 t} \bar{\omega}_{2 v}-\omega_{2 v} \bar{\omega}_{2}
\end{array}\right| .
\end{aligned}
$$

D'altra parte, in virtù delle (2) si ha

$$
\sum_{r, s}^{1 \ldots 2 p} \sum_{t, v}^{1 \ldots 2 p} c_{r s} c_{t v}\left(\omega_{1 r} \cdot \dot{\omega}_{2 s}-\omega_{18} \omega_{2 r}\right)\left(\bar{\omega}_{1 t} \bar{\omega}_{2 v}-\bar{\omega}_{1 v} \bar{\omega}_{2 t}\right)=0
$$

dunque, se indichiamo con $Q_{\text {rstv }}$ il determinante del $2^{0}$ ordine che appare nel penultimo sommatorio, possiamo anche scrivere:

$$
\varepsilon_{2}=-\frac{1}{4} \sum_{r, s}^{1 \ldots 2 p} \sum_{t, v}^{1 \ldots 2 p} c_{r s} c_{t v}\left[Q_{r s t v}-\left(\omega_{1 v} \cdot \omega_{2 s} \quad \omega_{1 s} \omega_{2 v}\right)\left(\bar{\omega}_{1 t} \cdot \bar{\omega}_{2 v}-\bar{\omega}_{1 v} \bar{\omega}_{2 t}\right)\right]
$$

Il coefficiente complessivo di $c_{r s} c_{t v}\left(^{1}\right)$ nello sviluppo del $2^{0}$ membro di questa nguaglianza è la somma dell'espressione chiusa qui sopra tra parentesi quadre o di quella che se ne ottiene scambiando $r$ con $t$ ed $s$ con $v$, oppure è la metri di questa somma secoudo che la coppia rs è o no distinta dalla coppia $t v$; ma una tal somma è lo sviluppo del determinante

$$
D_{r s t v}=\left|\begin{array}{llll}
\omega_{1 r} & \omega_{1 s} & \omega_{1 t} & \omega_{1 v} \\
\bar{\omega}_{1 r} & \bar{\omega}_{1 s} & \bar{\omega}_{1 t} & \bar{\omega}_{1 v} \\
\omega_{2 r} & \omega_{2 s} & \omega_{2 t} & \omega_{2 v} \\
\bar{\omega}_{2 r} & \bar{\omega}_{2 s} & \bar{\omega}_{2 t} & \bar{\omega}_{2 v}
\end{array}\right|
$$

(1) Si rioordi che si deve supporre $r<8$ e $t<v$. 
che è nullo se anche uno solo degli indici $r, s$ d uguale a uno degli indici $t$ e $v$, dunque quel coeficiente complessivo può considerarsi come dato in ogni caso da $D_{r s i v}$, e per conseguenza

$$
\varepsilon_{2}=-\frac{1}{4} \Sigma^{\prime \prime} c_{r s} c_{t v} D_{r s t v},
$$

dove i due apici apposti al simbolo del sommatorio stanno a significare che esso si estende a tutte le quadruple rstv di indici distinti scelti fra gli indici $1,2, \ldots, 2 p$, per le quali si ha $r<s$ e $t<v$, due di tali quadruple essendo da riguardarsi come identiche allorohè differiscono soltanto per l'ordine delle coppie rs e $t v$.

Siano $j_{1}, j_{2}, j_{3}, j_{4}$ quattro iudici scelti fra $1,2, \ldots, 2 p$ pei quali si abbia

$$
j_{1}<j_{2}<j_{3}<j_{4} .
$$

Allora nel sommatorio del secondo membro della (11) compaiono i termini

$$
c_{j_{1} j_{\mathrm{s}}} c_{j_{3} j_{4}} D_{j_{1} j_{2} j_{j} j_{4}}+c_{j_{1} j_{3}} c_{j_{2} j_{4}} D_{j_{1} j_{3} j_{2} j_{4}}+c_{j_{1} j_{4}} c_{j_{2} j_{3}} D_{j_{1} j_{4} j_{2} j_{3}}
$$

ma

$$
D_{j_{1} j_{j} j_{2} j_{4}}=-D_{j_{1} j_{2} j_{j} j_{4}}, \quad D_{j_{1} j_{4} j_{2} j_{3}}=D_{j_{1} j_{2 j} j_{4}}
$$

e inoltre, per le posizioni fatte nell'introduzione

$$
c_{j_{1} j_{2}} c_{j_{3} j_{4}}-c_{j_{1} j_{3}} c_{j_{2} j_{4}}+c_{j_{1} j_{4}} c_{j_{2} j_{3}}=P_{j_{1} j_{2} j_{j_{4}}},
$$

dunque la somma (12) pud scriversi

\section{$P_{j_{1} j_{2} j_{j} j_{4}} D_{j_{1} j_{2} j_{j} j_{4}}$}

Di qua, osservando che, per le solite posizioni,

$$
-D_{j_{1} j_{2} j_{3} j_{4}}=4 \delta_{j_{1} j_{2} j_{3} j_{4}},
$$

si trae finalmente, come volevasi,

$$
\varepsilon_{2}=-\Sigma P_{j_{1} j_{2} j_{3} j_{4}} \delta_{j_{1} j_{2} j_{3} j_{4}}=\Delta_{2} .
$$

4. Dimostriamo ora che

$$
\varepsilon_{h}=\Delta_{\Lambda} \quad(h=3,4, \ldots, p) .
$$


Ricordaudo il significato di $\varepsilon_{n}$ e sostituendo alle $A_{j k}$ i loro valori dati dalla (9) si trae

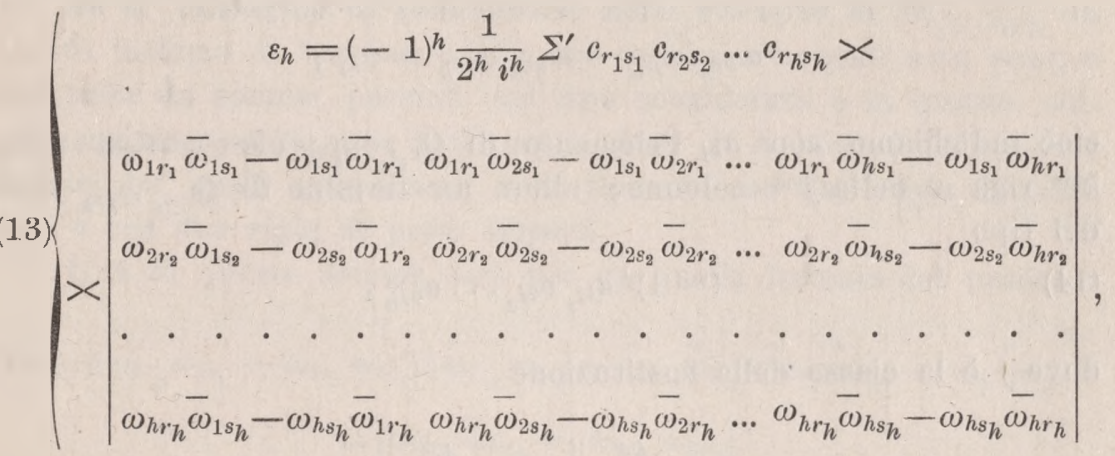

dove $\Sigma^{\prime}$ sta per

$$
\sum_{r_{1} s_{1}}^{1 \ldots 2 p} \sum_{r_{2} s_{2}}^{1 \ldots 2 p} \ldots \sum_{r_{h} s^{\prime}}^{\sum^{\prime}}
$$

Consideriamo il determinante che comparisce sotto il segno di sommatorio nel secondo membro della (13) e che per brevità chiameremo $Q_{r_{1} s_{1} \ldots r_{h} s_{h}}$; e poniamolo a raffronto col determinante

$$
D_{r_{1} s_{1} \ldots r_{h} s_{h}}=\left|\begin{array}{lllllll}
\omega_{1 r_{1}} & {\omega_{1 s_{1}}} & \dot{\omega}_{1 r_{2}} & \omega_{1 s_{2}} & \ldots & \omega_{1 r_{h}} & \omega_{1 s_{h}} \\
\bar{\omega}_{1 r_{1}} & \bar{\omega}_{1 s_{1}} & \bar{\omega}_{1 r_{2}} & \bar{\omega}_{1 s_{2}} & \ldots & \bar{\omega}_{1 r_{h}} & \bar{\omega}_{1 s_{h}} \\
\omega_{2 r_{1}} & \omega_{2 s_{1}} & \omega_{2 r_{2}} & \omega_{2 s_{2}} & \ldots & \omega_{2 r_{h}} & \omega_{2 s_{h}} \\
\bar{\omega}_{2 r_{1}} & \bar{\omega}_{2 s_{1}} & \bar{\omega}_{2 r_{2}} & \bar{\omega}_{2 s_{2}} & \ldots & \bar{\omega}_{2 r_{h}} & \bar{\omega}_{2 s_{h}} \\
\ldots & \ldots & \ldots & \ldots & \ldots & \ldots & \ldots \\
\omega_{h r_{1}} & \omega_{h s_{1}} & \omega_{h r_{2}} & \omega_{h s_{2}} & \ldots & \omega_{h r_{h}} & \omega_{h s_{h}} \\
\bar{\omega}_{h r_{1}} & \bar{\omega}_{h s_{1}} & \bar{\omega}_{h r_{2}} & \bar{\omega}_{h s_{2}} & \ldots & \bar{\omega}_{h r_{h}} & \bar{\omega}_{h s_{h}}
\end{array}\right| .
$$

Si scomponga la matrice di $D_{r_{1} s_{1} \ldots r_{h} s_{h}}$ in $h$ matrici rettangolari formate dalle colovne $1^{\mathrm{a}}$ e $2^{\mathrm{a}}, 3^{\mathrm{a}}$ e $4^{\mathrm{a}}, \ldots,(2 h-1)^{\mathrm{ma}}$ e $2 h^{\mathrm{ma}} ; \theta$ si immagini di sviluppare $D_{r_{1} s_{1} \ldots r_{h} s_{h}}$ per prodotti di minori (di $2^{0}$ ordine) contenuti in queste matrici secondo la regola generale di LAPLACE. 
Dico in primo luogo clie ogni termine del determinante $Q_{r_{1} s_{1} \ldots r_{h} h_{h}}$ è un termisue dello sviluppo di $D_{r_{1} s_{1} \ldots r_{h} s_{h}}$ così ottenuto.

Per comodita di scrittura poniamo

$$
\omega_{k r_{k}} \bar{\omega}_{j s_{k}}-\omega_{k s_{k}} \bar{\omega}_{j r_{k}}=a_{k j}
$$

cioè indichiamo con $a_{k j}$ l'elemento di $Q_{r_{1} s_{1} \ldots r_{h} s_{h}}$ che trovasi nella $k^{\text {ma }}$ riga e nella $j^{\text {pa }}$ colonna; allora un termine di $Q_{r_{1} s_{1} \ldots r_{h} s_{h}}$ sarà del tipo

$$
(-1)^{j} a_{1 j_{1}} a_{2 j_{2}} \ldots a_{h j_{h}}
$$

dove $j$ è la classe della sostituzione

$$
\left(\begin{array}{cccc}
j_{1} & j_{2} & \ldots & j_{h} \\
1 & 2 & \ldots & h
\end{array}\right)
$$

Che il termine (14), astrazion fatta dal segno, cornparisca nello sviluppo di $D_{r_{1} s_{1} \ldots r_{h} h}$ \& evidente; quindi tutto si riduce a far vedere che esso vi appare con lo stesso seguo.

Se uel prodotto (14), di cui ogni futtore è un binomio, si suppone di considerare il prodotto purziale formato coi primi termini dei binomi in discorso, si trova che questo è

$$
(-1)^{j} T=(-1)^{j} \omega_{1 r_{1}} \bar{\omega}_{j_{1} s_{1}} \omega_{2 r_{2}} \bar{\omega}_{j_{2} s_{2}} \ldots \omega_{h r_{h}} \bar{\omega}_{j_{h} s_{h}}
$$

Ora il segno di cui è affetto $T$ nello sviluppo ordinario di $D_{r_{1} s_{1} \ldots r_{h} s_{h}}$ è dato appunto, come subito si vede, da $(-1)^{j}$, dove $j$ è la classo della sostituzione (15), dunque la nostra asserzione è pienamente giustificata.

In secondo luogo si osservi che il valore di $D_{r_{1} s_{1} \ldots r_{h} s_{h}}$ nou muta se due qualnnque delle copple $r_{1} s_{1}, r_{2} s_{2}, \ldots, r_{h} s_{h}$ si scambiano fra loro; quindi lo sviluppo di $D_{r_{1} s_{1} \cdots r_{n} s_{h}}$, ottennto nel modo indicato più sopra, può-immaginarsi come decomposto iu tante somme parziali di prodotti di minori del $2^{0}$ ordine ognuna delle quali sia tale che tutti i suoi termini possano dedursi da uno qualunque di essi effettuando su questo ma qualunque permutazione fra le coppie $r_{1} s_{1}, r_{2} s_{2}, \ldots, r_{h} s_{h}$.

Un insieme di queste somme parziali sarà costituito da $Q_{r_{1} s_{1} \ldots r_{h} s_{h}}$ e dai determinanti che risultano da $Q_{r_{1} s_{1} \ldots r_{h} s_{h}}$ scambiaudo fra loro le coppie $r_{1} s_{1}, \ldots, r_{h} s_{h}$; esso, si vede subito, contiene tutti e soli i termini del nostro sviluppo di $D_{r_{1} s_{1} \ldots r_{h} h_{h}}$ che si ottengono pren- 
dendo in ciascuna delle matrici più sopra indicate un minore che sia formato da una riga di posto dispari $\theta$ da una riga di posto pari.

Se si immagina di prescindere nello sviluppo di $D_{r_{1} s_{1} \ldots r_{h} s_{h}}$ da questo insieme di termini, quello dei termini rimanenti sarà sempre costituito da somme parziali del tipo considerato e in queste singole somme ogni termine sarà un prodotto di $h$ minori delle nostre matrici dei quali uno almeno sarà formato con due righe di posto pari o con due righe di posto dispari.

Una di queste sornme sart̀ per es. quella formata dal prodotto

$$
\begin{gathered}
\text { (16) }-\left(\omega_{1 r_{1}} \omega_{2 s_{1}}-\omega_{1 s_{1}} \omega_{2 r_{1}}\right)\left(\bar{\omega}_{1 r_{2}} \bar{\omega}_{2 s_{2}}-\bar{\omega}_{1 s_{2}} \bar{\omega}_{2 r_{2}}\right)\left(\bar{\omega}_{3 r_{3}} \bar{\omega}_{3 s_{3}}-\omega_{3 s_{3}} \bar{\omega}_{3 r_{3}}\right) \ldots \\
\ldots\left(\omega_{h r_{h}} \bar{\omega}_{h s_{h}}-\omega_{h s_{h}} \bar{\omega}_{h r_{h}}\right)
\end{gathered}
$$

- da tutti quelli che se ne ottengono scambiando fra loro in tutte le maniere possibili le coppie $r_{1} s_{1}, \ldots, r_{h} s_{h}$.

Ebbene da ciascuna di queste somme estragghiamo un termine e diciamo

$$
H_{1}, \quad H_{2}, \ldots, \quad \Pi_{l}, \ldots
$$

i termini estratti; per fissar le idee possiamo supporre che $H_{1}$ sia precisamente il prodotto (16).

In virtù delle (2) e di quelle che ne discendono

$$
\sum_{r, s}^{1 \ldots 2 p} c_{r s} \bar{\omega}_{j r} \bar{\omega}_{k s}=0 \quad(j, k=1,2, \ldots p ; j<k),
$$

scritte sotto la forma

$$
\sum_{r . s}^{1 \ldots 2 p} c_{r s}\left(\omega_{j r} \omega_{k s}-\omega_{j s} \omega_{k r}\right)=0 \quad \text { o } \sum_{r, s}^{1 \ldots 2 p} c_{r s}\left(\bar{\omega}_{j r} \bar{\omega}_{k s}-\bar{\omega}_{j s} \bar{\omega}_{k r}\right)=0,
$$

è chiaro intanto che pud porsi

$$
\begin{gathered}
\dot{\varepsilon}_{h}=(-1)^{h} \frac{1}{2^{h} i^{h}} \Sigma^{\prime} c_{r_{1} s_{1}} c_{r_{2} s_{2}} \ldots \\
\ldots c_{r_{h} s_{h}}\left[Q_{r_{1} s_{1} \ldots r_{h} s_{h}}+H_{1}+H_{2}+\ldots+H_{l}+\ldots\right]
\end{gathered}
$$

poichè ad esempio

$$
\begin{gathered}
\Sigma^{\prime} c_{r_{1} s_{1}} c_{r_{2} s_{2}} \ldots c_{r_{h} s_{h}} H_{1}= \\
=-\sum_{r_{1} s_{1}}^{1 \ldots 2 p} c_{r_{1} s_{1}}\left(\omega_{1 r_{1}} \omega_{2 s_{1}}-\omega_{i s_{1}} \omega_{2 r_{1}}\right){ }_{r_{2} s_{2}}^{\sum^{\prime} c_{r_{2} s_{2}}}\left(\bar{\omega}_{1 r_{2}} \bar{\omega}_{2 s_{2}}-\bar{\omega}_{1 s_{2}} \bar{\omega}_{2 r_{2}}\right) \ldots=0 ;
\end{gathered}
$$


dunque, se le coppie $r_{1} s_{1} \ldots r_{h} s_{h}$ sono tutte distinie, il coefficiente complessivo di

$$
c_{r_{1} s_{1}} c_{r_{2} s_{2}} \ldots c_{r_{h} s}
$$

nello sviluppo finale del sommatorio del secondo membro della (17) è

$$
I_{r_{1} s_{1} \ldots r_{h} s_{h}}
$$

se no, è una conveniente parte aliquota di $D_{r_{1} s_{1} \ldots r_{h} s_{h}}$. Ma poichè questo determinante è uullo uppena due degli indici $r_{1} s_{1} \ldots r_{h} s_{h}$ sono uguali, può dirsi che quel coefficiente complessivo è in ogni caso $D_{r_{1} s_{1} \ldots r_{h} s_{h}}$ possiamo scrivere

$$
\varepsilon_{h}=(-1)^{h} \frac{1}{2^{h} i^{h}} \Sigma^{\prime \prime} c_{r_{1} s_{1}} c_{r_{2} s_{2}} \ldots c_{r_{h} s_{h}} D_{r_{1} s_{1} \ldots r_{h} s_{h}}
$$

dove i due apici apposti al simbolo del sommatorio stanno a significare che esso deve intendersi esteso a tutte le $2 h$-ple $r_{1} s_{1} r_{2} s_{2} \ldots r_{h} s_{h}$ di indici distinti scelti nella serie $1,2, \ldots, 2 p$, per le quali si ha $r_{1}<s_{1}, r_{2}<s_{2}, \ldots, r_{h}<s_{h}$, due di tali $2 h$-ple essendo da rignardarsi come identiche allorchè differiscono soltunto per l'ordine delle coppie $r_{1} s_{1}, r_{2} s_{2}, \ldots, r_{h} s_{h}$.

Ora evidentemente

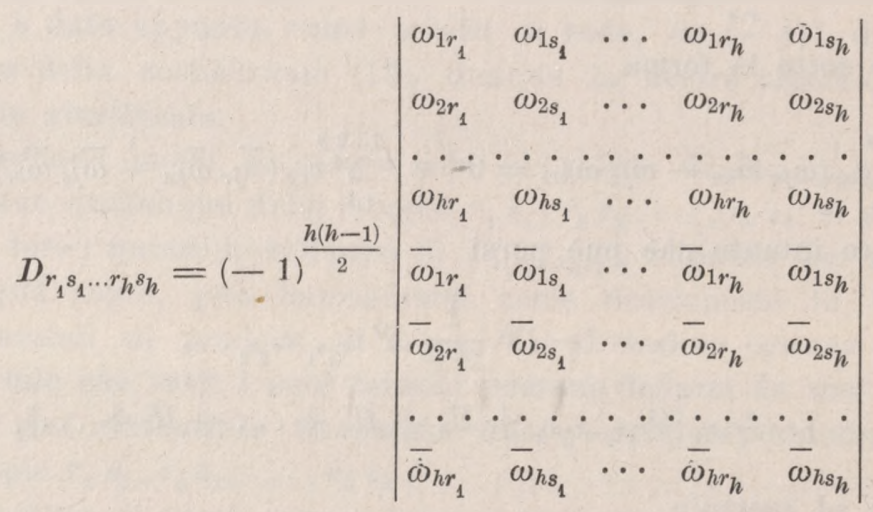

e il determinante che appare nel $2^{0}$ membro della (19) si riduce 
agevolmente $\left(^{1}\right)$ a

dunque

$$
(-1)^{h} 2^{h} i^{h} \delta_{r_{1} s_{1} \ldots r^{s} h}
$$

$$
\varepsilon_{h}=(-1)^{\frac{h(h-1)}{2}} \Sigma^{\prime \prime} c_{r_{1} s_{1}} c_{r_{2} s_{2}} \ldots c_{r_{h} s_{h}} \delta_{r_{1} s_{1} \ldots r_{h} s_{h}}
$$

Siano $j_{1}, j_{2}, \ldots, j_{2 h} 2 h$ indici scelti fra $1,2, \ldots, 2 p$ pei quali si abbia

$$
j_{1}<j_{2}<j_{3}<\ldots<j_{2 h} .
$$

Indicata con $\varrho_{1} \sigma_{1}, \varrho_{2} \sigma_{2}, \ldots, \varrho_{h} \sigma_{h}$ una qualunque distribuzione in coppie di codesti $2 h$ indici, il termine

$$
c_{\varrho_{1} \sigma_{1}} c_{\varrho_{2} \sigma_{2}} \cdots c_{\varrho_{h} \sigma_{h}}
$$

( $\left.{ }^{1}\right)$ Nel determinante in discorso si agginnga alla $1^{a}$ riga la $(h+1)^{\text {ma }}$, alla 2a riga la $(h+2)^{\mathrm{ma}}, \ldots$ alla $h^{\mathrm{ma}}$ riga l'ultima. Si vede $\cos$ che esso si riduce a

$$
2^{h}\left|\begin{array}{ccccc}
\alpha_{1 r_{1}} & \alpha_{1 s_{1}} & \ldots & \alpha_{1 r_{h}} & \alpha_{1 s_{h}} \\
\ldots & \ldots & \ldots & \ldots & \ldots \\
\alpha_{h r_{1}} & \alpha_{h s_{1}} & \ldots & \alpha_{h r_{h}} & \alpha_{h s_{h}} \\
\bar{\omega}_{1 r_{1}} & \bar{\omega}_{1 s_{1}} & \ldots & \bar{\omega}_{1 r_{h}} & \bar{\omega}_{1 s_{h}} \\
\ldots & \ldots & \ldots & \ldots & \ldots \\
\bar{\omega}_{h r_{1}} & \bar{\omega}_{h s_{1}} & \ldots & \bar{\omega}_{h r_{h}} & \bar{\omega}_{h s_{h}}
\end{array}\right|
$$

In quest'nltimo determinante si sottragga dalla $(h+1)^{\text {ma }}$ riga la $1 \mathrm{a}$, dalla $(h+2)^{\text {ma }}$ riga la $2 a, \ldots$ dall'ultima riga la $h^{\text {ma }}$; esso si couvertira in :

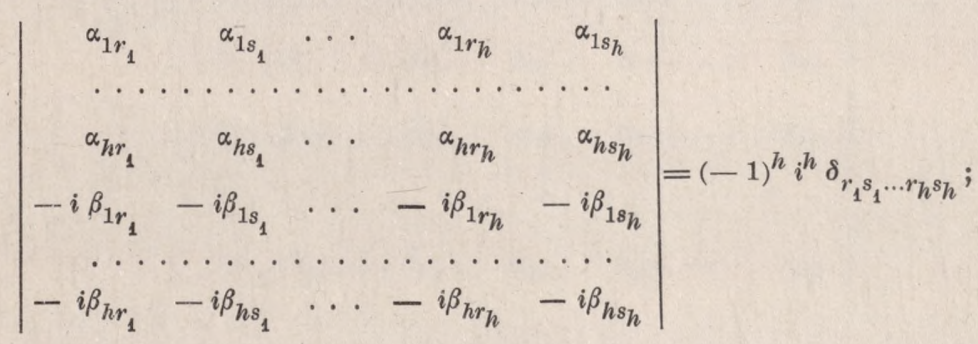

quindi la nostra affermazione è pienamente giustificata. 
apparisce nello pfaffiano

$$
P_{j_{1} j_{2} \ldots j_{2 h}}
$$

affetto dallo stesso segno che si deve attribuire a

$$
\delta_{j_{1} j_{2} \cdots j_{2 h}}
$$

perchè esso risulti uguale a

$$
\delta_{\varrho_{1} \sigma \cdots \varrho_{h} \sigma h}
$$

poichè l'uno e l'altro sono dati dal segno di $(-1)^{m}$, se $m$ è la classe della sostituzione

dunque, finulmente,

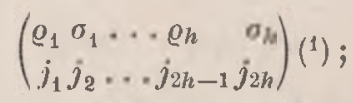

$$
\varepsilon_{h}=(-1)^{\frac{h(h-1)}{2}} \Sigma P_{j_{1} j_{2} \cdots j_{2 h}} \delta_{j_{1} j_{2} \cdots j_{2 h}}=\Delta_{h} .
$$

Cagliari, 10 gingno 1913.

(1) Vedi la mia Nota: Sui delerminanti emisimmetrici d'ordine pari e suoi relativi pfafiani [Rendioonti del Circolo Matematico di Palermo, t. XXXVI (20 semestre (1913), pp. 171-176]. 